\title{
Efeitos da Associação da Clonidina à Bupivacaína Hiperbárica na Anestesia Subaracnóidea Alta *
}

\section{Effects of Clonidine Associated to Hyperbaric Bupivacaine during High-Level Spinal Anesthesia}

José Reinaldo Cerqueira Braz, TSA ${ }^{1}$; Edgar Shiguero Koguti ${ }^{2}$; Leandro Gobbo Braz ${ }^{3}$; Lorena Brito da Justa Croitor, TSA ${ }^{2}$; Lais Helena Camacho Navarro ${ }^{3}$

\section{RESUMO}

Braz JRC, Koguti ES, Braz LG, Croitor LBJ, Navarro LHC - Efeitos da Associação da Clonidina à Bupivacaína Hiperbárica na Anestesia Subaracnóidea Alta

JUSTIFICATIVA E OBJETIVOS: Dados da literatura sugerem que a clonidina, um agonista $\alpha_{2}$-adrenérgico, em associação com a bupivacaína, na anestesia subaracnóidea alta pode aumentar a incidência de hipotensão e bradicardia. O objetivo desta pesquisa foi verificar o potencial sinergismo entre diferentes doses de clonidina, de 45 e $75 \mu \mathrm{g}$ e e bupivacaína hiperbárica nas características e nos efeitos hemodinâmicos do bloqueio subaracnóideo alto ( $\left.T_{4}\right)$.

MÉTODO: Em estudo aleatório e duplamente encoberto, foram avaliados 60 pacientes estado físico ASA I, submetidos à cirurgia do abdômen inferior e membros inferiores. Os pacientes foram submetidos à anestesia subaracnóidea, com bupivacaína hiperbárica a 0,5\% (17,5 mg - 3,5 ml) mais a associação das seguintes drogas: grupo Controle $(n=20)$ solução fisiológica $(0,5 \mathrm{ml})$; grupo Clon $45(n=20)$ - clonidina, na dose de $45 \mu \mathrm{g}(0,3 \mathrm{ml})$, associada à solução fisiológica $(0,2$ $\mathrm{ml})$; grupo Clon $75(n=20)$ - clonidina, na dose de $75 \mu \mathrm{g}(0,5$ $\mathrm{ml)}$. A cirurgia somente foi realizada quando o nível do dermátomo atingido pelo bloqueio analgésico foi de $T_{4}$.

RESULTADOS: A latência dos bloqueios analgésico e motor da anestesia subaracnóidea não diferiu significativamente entre os grupos $(p>0,05)$. Ambas as doses de clonidina prolongaram, de maneira significativa, a duração do bloqueio analgésico em $T_{8}$ e do bloqueio motor grau 3 (determinado pela escala modificada de Bromage) $(p<0,05)$. A incidência de hipotensão arterial no per-operatório foi evidente somente no grupo Clon 75 , em relação ao grupo Controle $(p<0,05)$, com o Clon 45 apresentando incidência intermediária entre os demais grupos. Não houve diferença significante entre os grupos em relação à bradicardia $(p>0,05)$. Ambas as doses de clonidina prolongaram a analgesia pós-operatória (intervalo de tempo decorrido entre o bloqueio subaracnóideo e a primeira

\footnotetext{
* Recebido do (Received from)CET/SBA do Departamento de Anestesiologia da Faculdade de Medicina de Botucatu (FMB - UNESP), Botucatu, SP

1. Professor Titular do CET/SBA do Departamento de Anestesiologia da FMB - UNESP

2. Anestesiologista do CET/SBA do Departamento de Anestesiologia da FMB - UNESP

3. $M E_{2}$ do CET/SBA do Departamento de Anestesiologia da FMB - UNESP
}

Apresentado (Submitted) em 24 de outubro de 2002

Aceito (Accepted) para publicação em 08 de janeiro de 2003

Endereço para correspondência (Correspondence to)

Dr. José Reinaldo Cerqueira Braz

Dept ${ }^{\circ}$ de Anestesiologia da FMB - UNESP

Distrito de Rubião Júnior

18618-970 Botucatu, SP

E-mail:jbraz@fmb.unesp.br

(C) Sociedade Brasileira de Anestesiologia, 2003 solicitação de analgésico pelo paciente no pós-operatório) ( $p<$ $0,05)$.

CONCLUSÕES: A clonidina na maior dose (75 $\mu \mathrm{g})$ em associação com a bupivacaína durante anestesia subaracnóidea alta $\left(T_{4}\right)$ determina maior incidência de hipotensão arterial, mas prolonga o bloqueio analgésico e a analgesia pós-operatória igualmente como a menor dose (45 $\mu \mathrm{g})$.

Unitermos: ANALGÉSICOS, clonidina; ANESTÉSICOS, Local: bupivacaína; TÉCNICAS ANESTÉSICAS, Regional: subaracnóidea

\section{SUMMARY}

Braz JRC, Koguti ES, Braz LG, Croitor LBJ, Navarro LHC Effects of Clonidine Associated to Hyperbaric Bupivacaine during High-Level Spinal Anesthesia

BACKGROUND AND OBJECTIVES: Published data suggest that clonidine, an $\alpha_{2}$-adrenergic agonist, in association with bupivacaine, may increase the incidence of intraoperative hypotension and bradycardia during high-level spinal anesthesia. This study aimed at determining the synergistic potential of two clonidine doses (45 and $75 \mu \mathrm{g}$ ) and hyperbaric bupivacaine on characteristics and hemodynamic effects of high-level ( $\left.T_{4}\right)$ spinal anesthesia.

METHODS: Participated in this randomized double-blind study, 60 ASA I patients scheduled for lower abdominal and limb surgery. Spinal anesthesia was induced with $17.5 \mathrm{mg}(3.5 \mathrm{ml})$ of $0.5 \%$ hyperbaric bupivacaine plus the association of the following drugs: Control group $(n=20)-0.5 \mathrm{ml}$ isotonic saline solution; Clon 45 group $(n=20)-45 \mu g(0.3 \mathrm{ml})$ clonidine $+0.2 \mathrm{ml}$ isotonic saline solution; and Clon $75(n=20)-75 \mu g(0.5 \mathrm{ml})$ clonidine. Surgery was only started when high-level $\left(T_{4}\right)$ analgesia was consistently obtained.

RESULTS: Sensory and motor block onset did not significantly differ among groups $(p>0.05)$. Both clonidine doses significantly prolonged analgesic block at $T_{8}$ and motor block level 3 duration (determined by modified Bromage scale) $(p<0.05)$. Intraoperative arterial hypotension was observed only in Clon 75 group as compared to Control group $(p<0.05)$, while Clon 45 group had intermediate incidence between both groups. There was no significant difference among groups in bradycardia ( $p>$ 0.05). Both clonidine doses prolonged postoperative analgesia (time from spinal block to first postoperative analgesic request) $(p<0.05)$.

CONCLUSIONS: High clonidine dose $(75 \mu \mathrm{g})$ associated to hyperbaric bupivacaine during high-level spinal anesthesia $\left(T_{4}\right)$ induces a higher incidence of arterial hypotension but prolongs sensory block and postoperative analgesia similar to lower clonidine dose (45 $\mu \mathrm{g})$ during upper spinal anesthesia.

Key Words: ANALGESICS: clonidine; ANESTHETICS: Local: bupivacaine; ANESTHETIC TECHNIQUES, Regional: spinal block 


\section{INTRODUÇÃO}

$\mathrm{D}$ urante a anestesia subaracnóidea, quando o nível do dermátomo atingido pelo bloqueio analgésico é alto $\left(T_{4}\right)$, os principais efeitos colaterais que podem ocorrer são as alterações cardiocirculatórias, como bradicardia e hipotensão arterial ${ }^{1}$. O bloqueio pré-ganglionar das fibras autonômicas vasoconstritoras produz vasodilatação arterial e venosa, com predomínio da venodilatação. Devido a esse fato, há diminuição do retorno venoso ao coração, com menor débito cardíaco, o qual, associado à diminuição da resistência vascular sistêmica, que é dependente do nível do dermátomo atingido pelo bloqueio, pode ocasionar hipotensão arterial. Já a freqüência cardíaca pode diminuir em razão do bloqueio das fibras cardioaceleradoras ( $\left.T_{1} a T_{4}\right)$, resultando numa predominância vagal, e das modificações das pressões intravasculares das grandes veias e do átrio direito, devido à diminuição do afluxo de sangue, com conseqüente ativação dos receptores intrínsecos de estiramento, que respondem com diminuição da freqüência cardíaca.

A clonidina, um agonista $\alpha_{2}$-adrenérgico, quando administrada por via subaracnóidea em associação com a bupivacaína, prolonga a duração da anestesia e da analgesia pós-operatória $^{2,7}$, mas pode aumentar a incidência de hipotensão arterial e bradicardia ${ }^{2,3-6,8}$. A clonidina, ao ativar os $\alpha_{2}$-receptores pós-sinápticos do núcleo do trato solitário e do locus cœruleus do tronco encefálico, diminui o efluxo simpático e as catecolaminas circulantes, com potencialização da atividade nervosa parassimpática, induzindo, dessa forma, redução da freqüência cardíaca e da pressão arterial ${ }^{9}$. Nas terminações nervosas periféricas, a clonidina inibe a exocitose da noradrenalina, determinando vasodilatação e redução do cronotropismo cardíaco. Essas ações explicam, parcialmente, o efeito hipotensor e bradicardizante dos agonistas desses receptores ${ }^{10}$. Existem também evidências que os efeitos hipotensivos dos $\alpha_{2}$-agonistas são aumentados pela inibição periférica da transmissão ganglionar que essas drogas determinam ${ }^{11}$.

O potencial sinergismo entre a clonidina e a anestesia subaracnóidea alta ainda não está bem estabelecido, principalmente em relação às alterações hemodinâmicas. quando em associação com a bupivacaína na anestesia subaracnóidea alta, determinem menores efeitos hemodinâmicos, o objetivo do estudo foi verificar o potencial sinergismo de doses menores da clonidina, nas doses de $45 \mu \mathrm{g}$ e $75 \mu \mathrm{g}$, com a bupivacaína hiperbárica nas características e nos efeitos hemodinâmicos do bloqueio subaracnóideo alto $\left(T_{4}\right)$.

\section{MÉTODO}

O estudo prospectivo, aleatório e duplamente encoberto foi realizado em 60 pacientes, de ambos os sexos, submetidos à cirurgia do abdômen inferior, períneo ou de membros inferiores. Todos os pacientes assinaram o termo de consentimento de participação, após receberem explicação detalhada do
Com a perspectiva de que doses menores de clonidina,

procedimento, o qual foi aprovado pelo Comitê de Ética local. Os critérios de exclusão foram idade maior do que 60 anos ou menor do que 18 anos, altura inferior a $155 \mathrm{~cm}$ e superior a $185 \mathrm{~cm}$ e índice de massa corporal superior a 30 .

Nenhum paciente recebeu medicação pré-anestésica. $\mathrm{Na}$ sala de operação foi feita a punção venosa com cateter $20 \mathrm{G}$ para administração de solução de Ringer com lactato (10 $\left.\mathrm{ml} \cdot \mathrm{kg}^{-1} \cdot \mathrm{h}^{-1}\right)$. A monitorização constou de pressão arterial não invasiva, determinada pelo método de Riva-Rocci, eletrocardioscopia na derivação $D_{\|}$, utilizada também para a medida da freqüência cardíaca, e oximetria de pulso para medida da saturação periférica de oxigênio da hemoglobina.

A punção subaracnóidea foi realizada com agulha de Quincke $26 G$, no espaço $L_{3}-L_{4}$, com os pacientes sentados. Os pacientes receberam, por via subaracnóidea, uma das seguintes combinações de drogas que foram preparadas por uma enfermeira, que não participou do estudo, após a abertura de envelope para identificação das drogas:

Grupo controle ( $\mathrm{n}=20$ ): bupivacaína hiperbárica - 17,5 mg $(3,5 \mathrm{ml})+$ solução fisiológica a $0,9 \%(0,5 \mathrm{ml})$.

Grupo Clon $45(n=20)$ : bupivacaína hiperbárica - $17,5 \mathrm{mg}$ $(3,5 \mathrm{ml})+$ solução fisiológica a $0,9 \%(0,2 \mathrm{ml})+$ clonidina $45 \mu \mathrm{g}(0,3 \mathrm{ml})$

Grupo Clon $75(\mathrm{n}=20)$ : bupivacaína hiperbárica - 17,5 mg $(3,5 \mathrm{ml})+$ clonidina $-75 \mu \mathrm{g}(0,5 \mathrm{ml})$.

Todos os pacientes receberam o mesmo volume das combinações das drogas no espaço subaracnóideo, ou seja, $3,5 \mathrm{ml}$ $+0,5 \mathrm{ml}$, em seringas separadas, na velocidade de $1 \mathrm{ml} 3 \mathrm{~s}^{-1}$. Após a realização do bloqueio subaracnóideo, os pacientes retornaram ao decúbito dorsal horizontal, colocando-se a mesa cirúrgica em cefalodeclive de $5 \mathrm{a} 10^{\circ}$ para que o bloqueio anestésico ficasse sempre em nível do dermátomo $\mathrm{T}_{4}$. Após o término da cirurgia, os pacientes foram encaminhados à Sala de Recuperação Pós-Anestésica (SRPA), onde permaneceram até a completa recuperação dos bloqueios motor e sensitivo, por um período máximo de seis horas após a realização do bloqueio subaracnóideo. Nesse período, a monitorização foi a mesma empregada na sala de operação. Quando o paciente queixava-se de dor e solicitava medicação analgésica, foi analisada a intensidade da dor pós-operatória pela Escala Visual Analógica de Dor, sendo então utilizada medicação analgésica do grupo dos antiinflamatórios não hormonais, por via venosa, com anotação desse tempo. Os atributos estudados foram:

- Bloqueio analgésico completo (tempo de latência): intervalo do tempo decorrido ( $\min$ ) entre o final da injeção do anestésico local $(A L)$ no espaço subaracnóideo e o aparecimento da analgesia cutânea progressiva nos dermátomos $T_{8}, T_{6}$ e $T_{4}$, pesquisado com auxílio de pinça anatômica na linha médio clavicular, bilateralmente, a cada minuto;

- Tempo de instalação e intensidade do bloqueio motor, de acordo com o critério proposto por Bromage ${ }^{12}$ (grau 0: capacidade em levantar os membros inferiores sem do- 
brar os joelhos; grau 1: capacidade em levantar os membros inferiores, dobrando os joelhos; grau 2: capacidade em flexionar apenas os pés e grau 3: imobilidade completa dos membros inferiores);

- Duração do bloqueio analgésico ( $\mathrm{min}$ ): desaparecimento da analgesia cutânea dois metâmeros $\left(T_{6}\right)$ e quatro metâmeros $\left(T_{8}\right)$ do nível analgésico $T_{4}$. Esse atributo foi avaliado a cada 10 minutos após 60 minutos da injeção do anestésico local no espaço subaracnóideo;

- Duração do bloqueio motor: baseado na capacidade do paciente em movimentar os membros inferiores, de acordo com o critério de Bromage. Esse atributo foi avaliado a cada 10 minutos após decorridos 60 minutos da injeção do anestésico local no espaço subaracnóideo;

- Nível de consciência: realizado de acordo com a escala numérica proposta por Filos e col. ${ }^{13}$ : 1) acordado e nervoso; 2) acordado e calmo; 3) sonolento mas facilmente despertável; 4) sonolento com despertar difícil. Este atributo foi pesquisado após 30 minutos da realização do bloqueio subaracnóideo e antes da realização de analgesia ou sedação complementar;

- Necessidade de analgesia complementar no per-operatório, que foi realizada com fentanil $(50 \mu \mathrm{g})$ por via venosa;

- Necessidade de sedação complementar no per-operatório, que foi realizada com midazolam $\left(0,03 \mathrm{mg} \cdot \mathrm{kg}^{-1}\right)$ por via venosa;

- Ocorrência de hipotensão arterial no per e pós-operatório, avaliada de acordo com o critério: igual ou acima de $30 \%$ da pressão arterial sistólica (PAS) inicial ou PAS inferior a $90 \mathrm{mmHg}$. As hipotensões foram tratadas inicialmente pelo aumento da infusão da solução Ringer com lactato e, quando necessário, por sulfato de efedrina (5 a $10 \mathrm{mg}$ ) por via venosa;

- Ocorrência de bradicardia no per e pós-operatório, avaliada de acordo com o critério: freqüência cardíaca menor do que 50 batimentos por minuto (bpm). Nas situações em que a freqüência cardíaca foi menor ou igual a 45 bpm ou quando houve instabilidade hemodinâmica, empregou-se sulfato de atropina $(0,5 \mathrm{mg})$ por via venosa;

- Duração da analgesia na SRPA: intervalo de tempo (min) decorrido entre o final da injeção do AL no espaço subaracnóideo e a primeira solicitação de analgésico pelo paciente;

- Intensidade da dor pós-operatória avaliada de acordo com a Escala Visual Analógica de Dor ( 0 = ausência de dor; 10 = dor máxima) no momento da primeira solicitação de analgesia pelo paciente. Nos pacientes que não solicitaram analgesia foi aplicada a escala de dor no momento da alta da SRPA;

- Efeitos colaterais foram anotados no per e pós-operatório: tremor, náuseas, vômitos, hipoxemia $\left(\mathrm{SpO}_{2} \leq 90 \%\right)$ e depressão respiratória (freqüência respiratória $\leq 10$ irpm).
Na análise estatística, para as variáveis idade, peso, altura e índice de massa corporal, utilizou-se a Análise de Variância. Para as variáveis mensuradas em escala ordinal, na comparação entre mais de dois grupos independentes, utilizou-se o teste não paramétrico de Kruskal-Wallis, seguido da comparação múltipla de Student-Newman-Keuls. Para a comparação das proporções, utilizou-se o teste Exato de Fisher ou o Qui-quadrado $\left(\mathrm{X}^{2}\right)$. As estatísticas foram consideradas significantes quando $p \leq 0,05$.

\section{RESULTADOS}

Os grupos estudados não diferiram quanto aos dados demográficos e sexo (Tabela I).

Tabela I - Dados Demográficos (Média $\pm D^{*}$ *) e Distribuição dos Sexos nos Grupos Estudados

\begin{tabular}{lccc}
\hline Variáveis & \multicolumn{3}{c}{ Grupos } \\
\cline { 2 - 4 } & $\begin{array}{r}\text { Controle } \\
(\mathrm{n}=20)\end{array}$ & $\begin{array}{c}\text { Clon 45 } \\
(\mathrm{n}=20)\end{array}$ & $\begin{array}{c}\text { Clon 75 } \\
(\mathrm{n}=20)\end{array}$ \\
\hline Idade $(\mathrm{anos})^{*}$ & $37 \pm 13$ & $35 \pm 12$ & $33 \pm 10$ \\
Peso $(\mathrm{kg})^{*}$ & $69 \pm 10$ & $69 \pm 16$ & $66 \pm 10$ \\
Altura $(\mathrm{cm}){ }^{*}$ & $168 \pm 8$ & $168 \pm 9$ & $166 \pm 10$ \\
Índice de massa corporal * $^{*}$ & $24,3 \pm 2,9$ & $24,2 \pm 4,1$ & $24,1 \pm 2,7$ \\
Sexo (n) & & & 12 \\
$\quad$ Masculino & 14 & 10 & 8 \\
\hline Feminino & 6 & 10 & 12 \\
\hline
\end{tabular}

Não houve diferença significativa entre os grupos $(p>0,05)$

As cirurgias realizadas nos três grupos estão apresentadas na tabela II.

Tabela II - Distribuição das Cirurgias nos Grupos Estudados

\begin{tabular}{lccc}
\hline & \multicolumn{3}{c}{ Grupos } \\
\cline { 2 - 4 } & Controle & Clon 45 & Clon 75 \\
\hline Herniorrafia inguinal & 8 & 6 & 6 \\
Apendicectomia & 1 & 1 & 1 \\
Colpoperineoplastia & 4 & 4 & 6 \\
$\begin{array}{l}\text { Safenectomia + retirada de trajetos } \\
\text { venosos }\end{array}$ & 1 & 3 & 1 \\
$\begin{array}{l}\text { Reconstrução de lesão do joelho } \\
\begin{array}{l}\text { Autoenxertia cutânea em membros } \\
\text { inferiores }\end{array}\end{array}$ & 1 & 2 & 2 \\
$\begin{array}{l}\text { Osteossíntese de fratura maleolar } \\
\text { de tornozelo }\end{array}$ & 3 & 3 & 2 \\
\hline
\end{tabular}

Com relação ao tempo de latência dos bloqueios sensitivo e motor, não houve diferença significativa entre os grupos (Tabela III). Também não houve diferença significativa entre os grupos em relação à intensidade do bloqueio motor, que ocorreu em grau 3 em todos os pacientes do grupo controle e em $95 \%$ dos pacientes dos demais grupos. 
Quanto à duração do bloqueio analgésico, houve diferença significativa entre os grupos (Tabela IV). Os grupos que receberam clonidina em relação ao controle, apresentaram aumento médio de $25 \%$ (Clon 75 ) a 27,5\% (Clon 45) na duração do bloqueio analgésico no dermátomo $T_{8}$. A duração do bloqueio motor grau 3 também foi significantemente maior nos grupos que receberam clonidina (15\% em Clon 45 e $25 \%$ em Clon 75) (Tabela IV).

Não houve diferença significativa entre os grupos em relação ao nível de consciência, com a maioria dos pacientes dos três grupos apresentando escore 2 (acordado e calmo) ( $p=$ 0,336).

Em relação à necessidade de analgesia complementar e de sedação no per-operatório, não houve diferença significativa entre os grupos, com pequeno número de pacientes (de um a três em cada grupo) que necessitaram de analgesia ou de sedação complementar nos grupos.

Na comparação da intensidade da dor na SRPA, houve diferença significativa entre os grupos $(p=0,022)$, com menores valores nos grupos que receberam clonidina (Figura 1). Aduração da analgesia no pós-operatório também foi significantemente maior nos grupos que receberam clonidina $(p=$ 0,008 ) (Figura 2). O número de pacientes que necessitou de analgesia no pós-operatório foi significativamente menor nos grupos que receberam clonidina (Tabela $\mathrm{V}$ ).

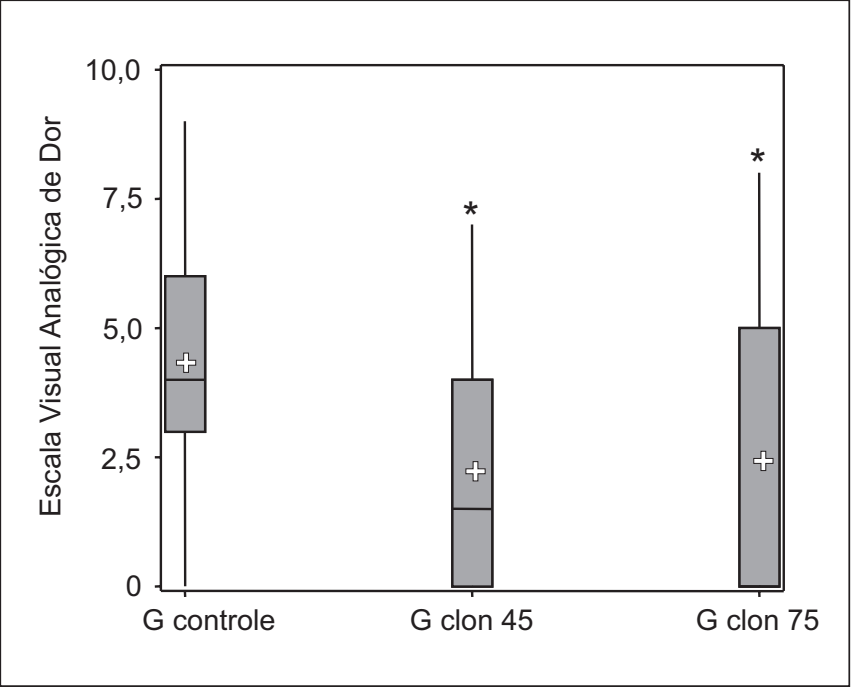

Figura 1 - Valores Médios, Superiores, Inferiores, da Mediana e do $1^{\circ}$ e $3^{\circ}$ Quartis da Escala Visual Analógica de Dor nos Grupos Estudados

${ }^{*} p<0,05$ em relação ao $G$ controle

Tabela III - Tempo (segundos) de Instalação dos Bloqueios Analgésico e Motor, com Indicação dos Valores da Mediana, $1^{\circ}$ e $3^{\circ}$ Quartis

\begin{tabular}{|c|c|c|c|}
\hline \multirow[t]{2}{*}{ Atributos } & \multicolumn{3}{|c|}{ Grupos } \\
\hline & Controle & Clon 45 & Clon 75 \\
\hline \multicolumn{4}{|c|}{ Bloqueio analgésico completo (s) } \\
\hline Dermátomo $T_{8}$ & $180(120-235)$ & $150(127-215)$ & $130(89-180)$ \\
\hline Dermátomo $\mathrm{T}_{6}$ & $210(169-327)$ & $215(166-285)$ & $182(135-220)$ \\
\hline Dermátomo $\mathrm{T}_{4}$ & $300(207-485)$ & $269(215-370)$ & $269(194-348)$ \\
\hline \multicolumn{4}{|c|}{ Instalação do bloqueio motor (s) } \\
\hline Grau 1 & $140(120-230)$ & $142(120-185)$ & $120(62-186)$ \\
\hline Grau 2 & $215(165-292)$ & $205(169-254)$ & $212(110-276)$ \\
\hline Grau 3 & $365(227-797)$ & $330(255-405)$ & $346(184-428)$ \\
\hline
\end{tabular}

Não houve diferença significante entre os grupos $(p>0,05)$ (Kruskal-Wallis)

Tabela IV - Duração dos Bloqueios Analgésico e Motor com Indicação dos Valores da Mediana, $1^{\circ}$ e $3^{\circ}$ Quartis

\begin{tabular}{lccc}
\hline Atributos & \multicolumn{3}{c}{ Grupos } \\
\cline { 2 - 4 } & Controle & G Clon 45 & G Clon 75 \\
\hline Duração do bloqueio analgésico (min) & & $148(107-190)$ & $145(120-180)$ \\
$\quad$ Dermátomo T6 & $115(95-148)$ & $189^{*}(127-217)$ & $183^{*}(147-210)$ \\
$\quad$ Dermátomo T8 & $137(110-171)$ & $252(195-300)$ \\
Duração do bloqueio motor (min) & & $215(172-274)$ & $226(170-280)$ \\
$\quad$ Grau 1 & $200(150-232)$ & $213(147-257)$ & $190^{*}(157-240)$ \\
Grau 2 & $197(100-230)$ & $168^{*}(150-210)$ & \\
Grau 3 & $142(117-179)$ & & \\
\hline
\end{tabular}

* Diferença significante entre os grupos tratados com clonidina e o grupo controle $(p<0,05)$ (Kruskal-Wallis) 


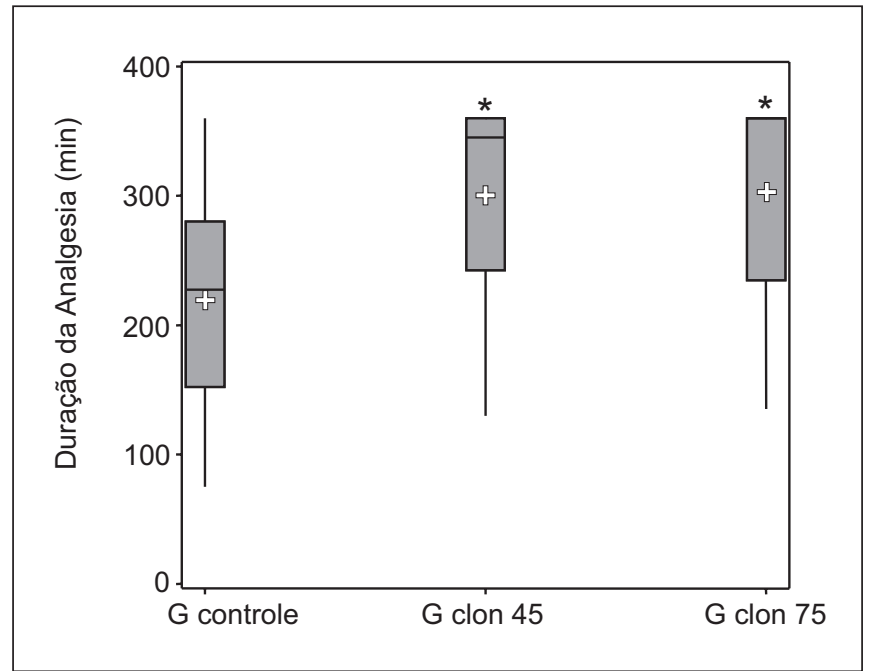

Figura 2 - Valores Médios, Superiores, Inferiores, da Mediana e do $1^{\circ}$ e $3^{\circ}$ Quartis da Duração da Analgesia Pós-Operatória nos Grupos Estudados

${ }^{*} p<0,05$ em relação ao $G$ controle

Tabela V - Distribuição de Pacientes que Necessitaram de Analgesia Complementar na SRPA nos Grupos Estudados

\begin{tabular}{lccc}
\hline Atributo & \multicolumn{3}{c}{ Grupos } \\
\hline & Controle & Clon 45 & Clon 75 \\
\hline $\begin{array}{l}\text { Necessidade de analgesia } \\
\text { complementar }\end{array}$ & $17 / 20$ & $10 / 20^{*}$ & $9 / 20^{*}$ \\
\hline
\end{tabular}

*Houve diferença significante em relação ao grupo controle $(p=0,019)$ (teste do $\mathrm{X}^{2}$ )

A ocorrência de bradicardia no per e pós-operatório foi pequena e os grupos não diferiram em sua distribuição (tabela $\mathrm{VI}$ ), mas houve diferença significante quanto à ocorrência de hipotensão arterial no per-operatório, com maior número de pacientes do grupo que recebeu a maior dose de clonidina apresentando esse evento em relação ao grupo controle, enquanto os pacientes que receberam a menor dose de clonidina apresentaram incidência de hipotensão arterial intermediária entre os dois grupos (Tabela VI).

A ocorrência de efeitos colaterais no per e pós-operatório foi pequena, sem diferença significativa entre os grupos (Tabelas VII e VIII).
Tabela VII - Ocorrência de Efeitos Colaterais no Per-Operatório nos Grupos Estudados

\begin{tabular}{lccc}
\hline Efeitos Colaterais & \multicolumn{3}{c}{ Grupos } \\
\cline { 2 - 4 } & Controle & Clon 45 & Clon 75 \\
\hline Tremor & $2 / 20$ & $3 / 20$ & $2 / 20$ \\
Náusea & $1 / 20$ & $3 / 20$ & $2 / 20$ \\
Vômito & $0 / 20$ & $1 / 20$ & $0 / 20$ \\
Hipoxemia & $0 / 20$ & $0 / 20$ & $1 / 20$ \\
\hline
\end{tabular}

Não houve diferença significante entre os grupos (teste do $\mathrm{X}^{2}$ )

Tabela VIII - Ocorrência de Efeitos Colaterais no Pós-Operatório nos Grupos Estudados

\begin{tabular}{lccc}
\hline Efeitos Colaterais & \multicolumn{3}{c}{ Grupos } \\
\cline { 2 - 4 } & Controle & Clon 45 & Clon 75 \\
\hline Tremor & $0 / 20$ & $0 / 20$ & $0 / 20$ \\
Náusea & $1 / 20$ & $0 / 20$ & $1 / 20$ \\
Vômito & $1 / 20$ & $0 / 20$ & $0 / 20$ \\
Hipoxemia & $1 / 20$ & $0 / 20$ & $0 / 20$ \\
\hline
\end{tabular}

Não houve diferença significante entre os grupos (Teste do $X^{2}$ )

\section{DISCUSSÃO}

Na presente pesquisa, a incidência de hipotensão arterial foi importante e significativa quando se obteve nível alto da anestesia subaracnóidea e empregou-se a maior dose de clonidina $(75 \mu \mathrm{g})$. Já com a menor dose de clonidina $(45 \mu \mathrm{g})$, a incidência de hipotensão arterial foi intermediária, sem diferença significativa com o grupo controle ou com o grupo Clon 75.

Em adição às ações periféricas e no tronco cerebral, a administração raquidiana da clonidina inibe os neurônios pré-ganglionares simpáticos da medula espinhal ${ }^{14}$. Assim, a intensidade da hipotensão arterial, induzida pela clonidina, por via espinhal, está relacionada ao nível do dermátomo onde é administrada ${ }^{15}$. Em níveis torácico baixo e lombar, a administração peridural de clonidina não aumenta a incidência de hipotensão arterial ${ }^{15}$, mas quando a administração é feita em nível torácico alto, observa-se incidência aumentada desse efeito ${ }^{16}$. Em bloqueios subaracnóideos mais bai$\operatorname{xos}\left(\mathrm{T}_{10} \mathrm{a} \mathrm{T}_{6}\right)$, a incidência de hipotensão arterial com a clonidina, em doses de 75 a $225 \mu$ g, é de $20 \%{ }^{2,3,6}$. Por outrolado, a ocorrência de hipotensão é maior nos pacientes com doença hipertensiva arterial do que nos que apresentam pressão ar-

Tabela VI - Ocorrência de Hipotensão Arterial e Bradicardia no Per e Pós-Operatório nos Grupos Estudados

\begin{tabular}{lcccccc}
\hline \multirow{2}{*}{ Atributos } & \multicolumn{3}{c}{ Grupos } \\
\cline { 2 - 7 } & \multicolumn{2}{c}{ Controle } & \multicolumn{2}{c}{ Clon 45 } & \multicolumn{2}{c}{ Clon 75} \\
\cline { 2 - 7 } & Per-Operatório & Pós-Operatório & Per-Operatório & Pós-Operatório & Per-Operatório & Pós-Operatório \\
\hline Hipotensão arterial & $2 / 20$ & $0 / 20$ & $5 / 20$ & $1 / 20$ & $9 / 20 *$ & $1 / 20$ \\
Bradicardia & $2 / 20$ & $0 / 20$ & $3 / 20$ & $1 / 20$ & $3 / 20$ & $1 / 20$ \\
\hline
\end{tabular}

* Diferença estatisticamente significante em relação ao grupo controle $(p<0,030)$ (Kruskal-Wallis) 
terial em níveis normais, após a administração sistêmica ou raquídea da clonidina refletindo o aumento do tônus simpático nesses pacientes ${ }^{8}$.

Apesar de o conjunto de evidências, que relaciona a ativação dos receptores $\alpha_{2}$-adrenérgicos com o efeito hipotensor e de bradicardia dos agonistas desses receptores, não se exclui a participação dos receptores imidazolínicos $I_{1}$ situados no núcleo reticular, cuja ativação provocada pela clonidina, que é um derivado imidazolínico, pode determinar bradicardia e hipotensão arterial ${ }^{17}$.

A hipotensão arterial por $\alpha_{2}$-agonista é facilmente corrigida pela administração de $\alpha_{1}$-adrenérgicos, como a efedrina, que apresenta resposta vasoconstritora aumentada na presença dessas drogas ${ }^{18}$

Já a incidência de bradicardia foi pequena, sem diferença significante entre os grupos e semelhante à encontrada também por outros autores ${ }^{6,7}$.

A pesquisa mostrou que a diminuição da dose de clonidina em associação à bupivacaína hiperbárica na anestesia subaracnóidea não interferiu com as principais vantagens que são obtidas com essa associação, como o aumento da duração do bloqueio sensitivo e da analgesia no pós-operatório, com menores valores da Escala Visual Analógica de Dor e menor número de pacientes necessitando analgesia complementar na SRPA, em relação ao grupo controle.

A associação também aumentou a duração do bloqueio motor grau 3 , embora em tempo menor (15\% a $25 \%$ ) do que quando se utiliza essa droga em doses superiores a $75 \mu \mathrm{g}$, que é de $30 \%$ a $35 \%{ }^{8}$. Deve-se ressaltar que o aumento da duração do bloqueio motor, que pode ser importante em cirurgias que necessitam bom relaxamento muscular, é sempre um fator de preocupação e de desconforto para os pacientes no pós-operatório. Além disso, não houve aumento significante do tempo de regressão dos bloqueios motor graus 2 e 1 , com as duas doses de clonidina.

Embora já tenha sido bem comprovado que a clonidina prolonga a duração dos bloqueios sensitivo, motor e analgésico da bupivacaína na anestesia subaracnóidea ${ }^{2,7}$, o mecanismo desses efeitos ainda não está bem estabelecido.

Assim, pesquisas experimentais ${ }^{19,21}$ e clínicas ${ }^{13,22}$ demonstraram que a clonidina administrada por via peridural ou subaracnóidea apresenta potente ação analgésica, ao ativar os $\alpha_{2}$-adrenorreceptores da substância cinzenta da coluna dorsal da medula espinhal. Esta ação antinociceptiva ocorre tanto para a dor somática ${ }^{19}$ como para a visceral ${ }^{23}$.

Alguns investigadores ${ }^{7}$ sugerem também uma ação anestésica local própria da clonidina, o que tem propiciado a associação da droga com anestésicos locais em bloqueios raquídeos. Essa ação da clonidina, semelhante à do anestésico local, e a interação com anestésicos locais têm sido explicadas por três possíveis mecanismos. Primeiro, a clonidina bloqueia a condução pelas fibras $A \delta$ e $C$, como conseqüência do aumento da condutância de potássio em neurônios isolados e, portanto, intensifica o bloqueio de condução dos anestésicos locais ${ }^{24}$. Segundo, a clonidina pode causar vasoconstrição local diminuindo, conseqüentemente, a dispersão e remoção local do anestésico local em torno das estrutu- ras neurais, efeito mediado pela ação da droga nos $\alpha_{2}$-receptores pós-sinápticos, embora as evidências sejam pequenas para esse mecanismo, com as doses clínicas utilizadas $^{25}$. Terceiro, quando a clonidina é associada a anestésicos locais para aplicação espinhal, como na presente pesquisa, ou em bloqueios periféricos, intensifica e prolonga a ação analgésica ${ }^{26}$. No tratamento da dor aguda, a clonidina por via subaracnóidea é duas vezes mais potente do que por via peridural ${ }^{27}$.

Os agonistas $\alpha_{2}$-adrenérgicos, quando utilizados por via subaracnóidea, também podem causar analgesia, em parte, por ativação de neurônios colinérgicos espinhais, resultado da liberação de acetilcolina ${ }^{28}$.

A clonidina, nas doses empregadas, não provocou efeito sedativo significante, que também parece não ter ocorrido quando a droga foi empregada em doses maiores $(150 \mu \mathrm{g})$ por via subaracnóidea ${ }^{6,7}$. Esses resultados contrastam com os obtidos pelos autores quando a clonidina foi empregada por via oral $(150 \mu \mathrm{g})^{29,30}$ ou peridural $(300 \mu \mathrm{g})^{31}$, quando o efeito sedativo da droga foi evidente. O locus cœrulus é a principal região do sistema nervoso central envolvida com o efeito sedativo dos agonistas dos receptores $\alpha_{2}$-adrenérgi$\cos$. As principais vias adrenérgicas ascendentes e descendentes originam-se dessa área. A ativação dos $\alpha_{2}$-receptores dessa área produz supressão de sua atividade, resultando em aumento da atividade de interneurônios inibitórios, como o da via do ácido $\gamma$-aminobutírico (GABA), o que determina depressão do SNC ${ }^{32}$.

Nas condições deste estudo, o emprego da clonidina nas doses de 45 e 75 g em associação com a bupivacaína hiperbárica durante anestesia subaracnóidea alta, atingindo o dermátomo $\mathrm{T}_{4}$, mantém as principais vantagens da associação, que são o aumento da duração do bloqueio analgésico e da analgesia no pós-operatório. No entanto, a maior dose $(75$ $\mu \mathrm{g})$ da clonidina aumenta significantemente a incidência de hipotensão arterial.

\section{Effects of Clonidine Associated to Hyperbaric Bupivacaine during High-Level Spinal Anesthesia}

José Reinaldo Cerqueira Braz, TSA, M.D.; Edgar Shiguero Koguti, M.D.; Leandro Gobbo Braz, M.D.; Lorena Brito da Justa Croitor, TSA, M.D.; Lais Helena Camacho Navarro, M.D.

\section{INTRODUCTION}

When the dermatome level reached by analgesic block is high $\left(\mathrm{T}_{4}\right)$ during spinal anesthesia, major side-effects are cardiocirculatory changes, such as bradycardia and arterial hypotension ${ }^{1}$. Pre-ganglial block of vasoconstricting autonomic fibers causes arterial and venous vasodilatation, with predominance of venodilatation. Therefore, there is a ve- 
nous return decrease to heart with lower cardiac output associated to systemic vascular resistance decrease, which is dependent on the dermatome reached by the blockade. Those modifications may cause arterial hypotension. Heart rate may decrease as a function of cardioaccelerator fibers block ( $T_{1}$ to $\left.T_{4}\right)$, resulting in vagal predominance, and of great vessels and right atrium intravascular pressure changes due to decreased blood flow, with the consequent activation of stretching intrinsic receptors which respond by decreasing heart rate.

Clonidine, an $\alpha_{2}$-adrenergic agonist, prolongs anesthesia and postoperative analgesia when associated to bupivacaine in spinal anesthesia ${ }^{2,7}$, but may increase the incidence of arterial hypotension and bradycardia ${ }^{2,3-6,8}$. In activating post-synaptic $\alpha_{2}$-receptors of the solitaire tract nucleus and of brain stem locus coeruleus, clonidine decreases sympathetic efflux and circulating catecholamines, with parasympathetic nervous activity potentiation, thus inducing heart rate and blood pressure decrease ${ }^{9}$. In peripheral nervous terminations, clonidine inhibits norepinephrine exocytosis, determining vasodilatation and heart chronotropism decrease. These actions partially explain hypotension and bradycardia induced by these receptors' agonists ${ }^{10}$.

There are also evidences that $\alpha_{2}$-agonists hypotensive effects are increased by the peripheral inhibition of ganglial transmission determined by such drugs ${ }^{11}$.

The potential synergism between clonidine and high-level spinal anesthesia is not well established, especially for hemodynamic changes.

With the perspective that lower clonidine doses associated to bupivacaine in high-level spinal anesthesia determine lower hemodynamic effects, this study aimed at observing the potential synergism of lower clonidine doses $(45 \mu \mathrm{g}$ and $75 \mu \mathrm{g})$ and hyperbaric bupivacaine in high-level $\left(T_{4}\right)$ spinal block hemodynamic effects.

\section{METHODS}

Participated in this prospective randomized double-blind study 60 patients of both genders, submitted to lower abdomen, perineal or lower limbs surgery. All patients gave their written consent after receiving detailed information on the procedure, which had been previously approved by the local Ethics Committee. Exclusion criteria were age above 60 years or below 18 years, height below $155 \mathrm{~cm}$ and above 185 $\mathrm{cm}$ and body mass index above 30 .

Patients were not premedicated. Venous puncture was performed in the operating room with a $20 \mathrm{G}$ catheter for lactated Ringer's infusion $\left(10 \mathrm{ml} \cdot \mathrm{kg}^{-1} \cdot \mathrm{h}^{-1}\right)$. Monitoring consisted of non-invasive blood pressure by the Riva-Rocci method, ECG

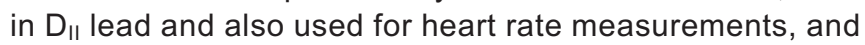
pulse oximetry for hemoglobin peripheral oxygen saturation. Spinal puncture was performed with 26G Quincke needle in $L_{3}-L_{4}$ interspace, with patients in the sitting position. One of the following drug combinations, prepared by a nurse not par- ticipating in the study and after the opening of the envelope to identify drugs, was spinally administered:

Control group $(\mathrm{n}=20)$ : hyperbaric bupivacaine $-17.5 \mathrm{mg}$ $(3.5 \mathrm{ml})+0.9 \%$ saline solution $(0.5 \mathrm{ml})$;

Clon 45 group $(n=20)$ : hyperbaric bupivacaine $-17.5 \mathrm{mg}$ $(3.5 \mathrm{ml})+0.9 \%$ saline solution $(0.2 \mathrm{ml})+$ clonidine $-45 \mu \mathrm{g}$ $(0.3 \mathrm{ml})$;

Clon $75(n=20)$ : hyperbaric bupivacaine $-17.5 \mathrm{mg}(3.5 \mathrm{ml})$ + clonidine - $75 \mu \mathrm{g}(0.5 \mathrm{ml})$.

All patients received the same volume of drug combinations in the spinal space, that is, $3.5 \mathrm{ml}+0.5 \mathrm{ml}$, in separate syringes, at a rate of $1 \mathrm{ml} .3 \mathrm{~s}^{-1}$. After induction, patients returned to the supine position and the table was placed in $5^{\circ}$ to $10^{\circ}$ head-down position for the anesthetic block to remain always at the level of $\mathrm{T}_{4}$.

At surgery completion, patients were referred to the Post-Anesthetic Recovery Unit (PACU) where they remained until total motor and sensory block recovery, for a maximum period of 6 hours after spinal block. During this period, monitoring was the same as in the operating room. Pain Analog Visual Scale evaluated postoperative pain intensity when patients referred pain and requested analgesic medication, which consisted of intravenous non-steroid anti-inflammatory drugs, being this moment also recorded.

Studied attributes were:

- Total analgesic block (onset): time elapsed ( $\mathrm{min}$ ) between end of local anesthetic (LA) spinal injection and progressive skin analgesia in dermatomes $T_{8}, T_{6}$ and $T_{4}$, evaluated with the aid of an anatomic forceps in the medium clavicular line, bilaterally, at 1-minute intervals;

- Motor block onset and intensity according to Bromage's criteria $^{12}$ (grade 0: ability to raise lower limbs without bending the knees; grade 1: ability to raise lower limbs bending the knees; grade 2: ability to bend feet only and grade 3: total lower limbs immobility;

- Analgesic block duration (min): recovery of skin analgesia in two metamers $\left(T_{6}\right)$ and four metamers $\left(T_{8}\right)$ of the analgesic level $\mathrm{T}_{4}$. This attribute was evaluated at $10-\mathrm{mi}-$ nute intervals 60 minutes after local anesthetic spinal injection;

- Motor block duration: based on patients' ability to move lower limbs and according to Bromage's criteria. This attribute was evaluated at 10-minute intervals 60 minutes after local anesthetic spinal injection;

- Consciousness level: according to Filos et al. ${ }^{13}$ numerical scale: 1) awaken and nervous; 2) awaken and relaxed; 3) sleepy but easily awakened; 4) sleepy and difficult to awaken. This attribute was evaluated 30 minutes after spinal block and before additional analgesia or sedation;

- Need for additional intraoperative analgesia with intravenous fentanyl $(50 \mu \mathrm{g})$; 
- Need for additional intraoperative sedation with intravenous midazolam $\left(0.03 \mathrm{mg} \cdot \mathrm{kg}^{-1}\right)$;

- Intra and postoperative arterial hypotension, evaluated as equal to or above $30 \%$ of initial systolic blood pressure (SBP) or SBP lower than $90 \mathrm{mmHg}$. Arterial hypotension was initially treated by increasing lactated Ringer's solution and, when needed, with intravenous ephedrine (5 to $10 \mathrm{mg}$ );

- Intra and postoperative bradycardia evaluated as heart rate below 50 beats per minute (bpm). When heart rate was equal to or lower than $45 \mathrm{bpm}$ or when there was hemodynamic instability, intravenous atropine $(0.5 \mathrm{mg})$ was administered;

- Analgesia duration in the PACU: time elapsed (min) between end of LA spinal injection and first analgesic request by patients;

- Postoperative pain intensity evaluated by the Pain Ana$\log$ Visual Scale $(0=$ absence of pain; $10=$ maximum possible pain) when patients requested analgesic medication for the first time. Patients not requesting analgesics were submitted to the pain scale at PACU discharge;

- Intra and postoperative side-effects were recorded: shivering, nausea, vomiting, hypoxemia $\left(\mathrm{SpO}_{2} \leq 90 \%\right)$ and respiratory depression (respiratory rate $\leq 10 \mathrm{irpm}$ ).

For statistical analysis, Analysis of Variance was used to compare age, weight, height and body mass index. For variables measured in ordinal scale to compare more than two independent groups, Kruskal-Wallis non-parametric test was used, followed by Student-Newman-Keuls multiple comparisons. Fisher's Exact test or Chi-square test $\left(X^{2}\right)$ were used to compare proportions. Statistics were considered significant when $p \leq 0.05$.

\section{RESULTS}

There were no differences among groups in demographics data and gender (Table I).

There has been no significant differences among groups in sensory and motor block onset (Table III). There has also been no significant differences among groups in motor block intensity, which has reached level 3 in all control group patients and in $95 \%$ of remaining groups patients.
Table I - Demographics Data (Mean \pm SD*) and Gender Distribution within Studied Groups

\begin{tabular}{lccc}
\hline Variables & \multicolumn{3}{c}{ Groups } \\
\cline { 2 - 4 } & $\begin{array}{c}\text { Control } \\
(\mathrm{n}=20)\end{array}$ & $\begin{array}{c}\text { Clon 45 } \\
(\mathrm{n}=20)\end{array}$ & $\begin{array}{c}\text { Clon 75 } \\
(\mathrm{n}=20)\end{array}$ \\
\hline Age (years) ${ }^{*}$ & $37 \pm 13$ & $35 \pm 12$ & $33 \pm 10$ \\
Weight $(\mathrm{kg})^{*}$ & $69 \pm 10$ & $69 \pm 16$ & $66 \pm 10$ \\
Height $(\mathrm{cm})^{*}$ & $168 \pm 8$ & $168 \pm 9$ & $166 \pm 10$ \\
Body mass index ${ }^{*}$ & $24.3 \pm 2.9$ & $24.2 \pm 4.1$ & $24.1 \pm 2.7$ \\
Gender (n) & & & 12 \\
$\quad$ Male & 14 & 10 & 8 \\
\hline \multicolumn{1}{c}{ Female } & 6 & 10 & 12 \\
\hline
\end{tabular}

There were no significant differences among groups $(p>0.05)$

Table II shows surgeries performed in the three groups.

Table II - Surgeries Distribution among Groups

\begin{tabular}{lccc}
\hline Surgeries & \multicolumn{3}{c}{ Groups } \\
\cline { 2 - 4 } & Control & Clon 45 & Clon 75 \\
\hline Inguinal hernia & 8 & 6 & 6 \\
Appendicitis & 1 & 1 & 1 \\
Colpoperineoplasty & 4 & 4 & 6 \\
$\begin{array}{l}\text { Saphenectomy + venous } \\
\text { pathways removal }\end{array}$ & 1 & 3 & 1 \\
Knee reconstruction & 1 & 2 & 2 \\
Lower limb skin autografting & 2 & 1 & 2 \\
$\begin{array}{l}\text { Ankle maleolar fracture } \\
\text { osteosynthesis }\end{array}$ & 3 & 3 & 2 \\
\hline
\end{tabular}

There have been significant differences among groups in analgesic block duration (Table IV). Groups receiving clonidine had a mean $25 \%$ (Clon 75 ) and $27.5 \%$ (Clon 45 ) increase in analgesic block duration in dermatome $\mathrm{T}_{8}$, as compared to control group. Motor block level 3 was also significantly longer in groups receiving clonidine (15\% in Clon 45 and $25 \%$ in Clon 75) (Table IV).

Table III - Time (seconds) for Analgesic and Motor Block Installation, with indication of Median $1^{\text {st }}$ and $2^{\text {nd }}$ Quartiles

\begin{tabular}{lccc}
\hline Attributes & \multicolumn{3}{c}{ Groups } \\
\cline { 2 - 4 } & Control & Clon 45 & Clon 75 \\
\hline Total analgesic block (s) & & & $130(89-180)$ \\
$\quad$ Dermatome $T_{8}$ & $180(120-235)$ & $150(127-215)$ & $182(135-220)$ \\
$\quad$ Dermatome $T_{6}$ & $210(169-327)$ & $215(166-285)$ & $269(194-348)$ \\
$\quad$ Dermatome $T_{4}$ & $300(207-485)$ & $269(215-370)$ & $120(62-186)$ \\
Motor block installation (s) & & & $212(110-276)$ \\
Grade 1 & $140(120-230)$ & $205(169-254)$ & $346(184-428)$ \\
Grade 2 & $215(165-292)$ & $330(255-405)$ & \\
Grade 3 & $365(227-797)$ & & \\
\hline
\end{tabular}

There were no significant differences among groups $(p>0.05)$ (Kruskal-Wallis) 
Table IV - Duration of Analgesic and Motor Blocks with Indication of Median, $1^{\text {st }}$ and $2^{\text {nd }}$ Quartiles

\begin{tabular}{lccc}
\hline Attributes & \multicolumn{1}{c}{ Groups } & G Clon 75 \\
\cline { 2 - 4 } & Control & G Clon 45 & $145(120-180)$ \\
\hline $\begin{array}{l}\text { Analgesic block duration (min) } \\
\text { Dermatome } T_{6}\end{array}$ & $115(95-148)$ & $148(107-190)$ & $183^{*}(147-210)$ \\
$\quad$ Dermatome T8 & $137(110-171)$ & $189^{*}(127-217)$ & $252(195-300)$ \\
Motor block duration (min) & & & $215(172-274)$ \\
$\quad$ Grade 1 & $200(150-232)$ & $213(147-257)$ & $190-280)$ \\
Grade 2 & $197(100-230)$ & $168^{*}(150-210)$ & $190^{*}(157-240)$ \\
$\quad$ Grade 3 & $142(117-179)$ &
\end{tabular}

* Significant differences between clonidine-treated groups and control group $(p<0.05)$ (Kruskal-Wallis)

There were no significant differences among groups in consciousness level, with most patients in all groups presenting score 2 (awaken and relaxed) $(p=0.336)$. There have also been no significant differences among groups in the need for additional intraoperative analgesia or sedation, with a small number of patients (from one to three in each group), needing additional analgesia or sedation.

There have been significant differences among groups in pain intensity in the PACU $(p=0.022)$ with lower values for clonidine groups (Figure 1). Postoperative analgesia duration was also significantly longer in clonidine groups $(p=0.008)$ (Figure 2$)$. The number of patients needing postoperative analgesia was significantly lower in the groups receiving clonidine (Table V).

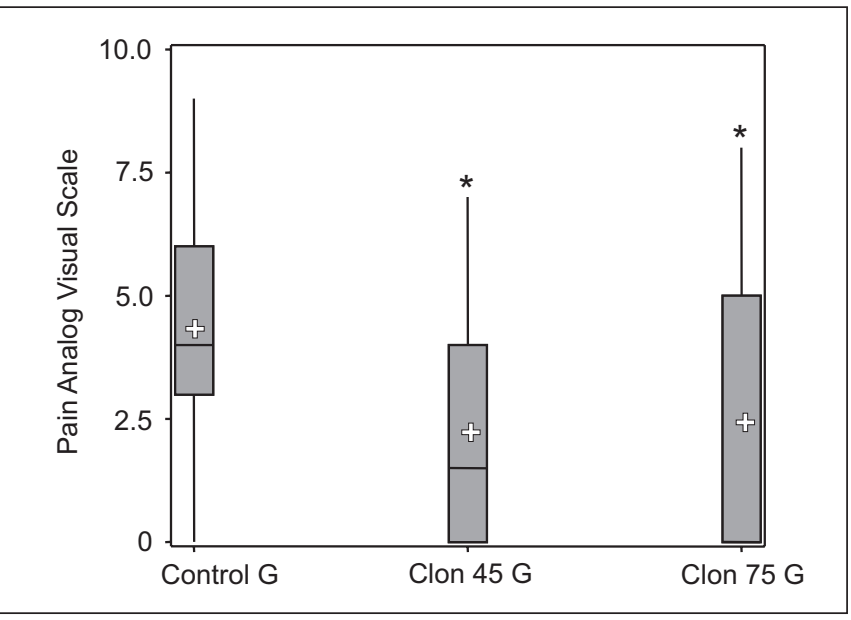

Figure 1 - Mean, Upper and Lower Values of Median, $1^{\text {st }}$ and $3^{\text {rd }}$ Quartiles of Pain Analog Visual Scale among Groups * $p<0.05$ as compared to Control G

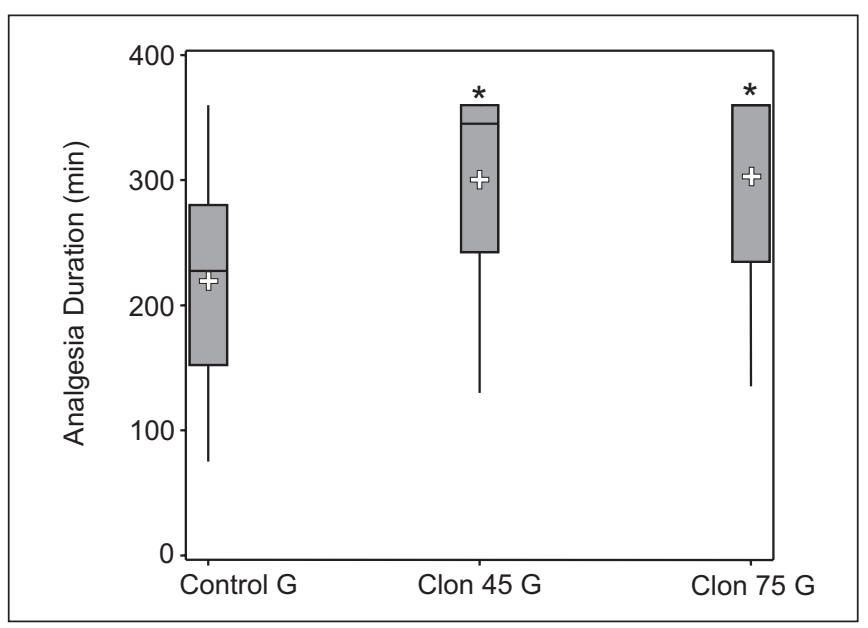

Figure 2 - Mean, Upper and Lower Values of Median, $1^{\text {st }}$ and $3^{\text {rd }}$ Quartiles of Postoperative Analgesia Duration among Groups ${ }^{*} p<0.05$ as compared to Control G

Table V - Distribution of Patients Needing Additional Analgesic in the PACU

\begin{tabular}{lccc}
\hline Attributes & \multicolumn{3}{c}{ Groups } \\
\cline { 2 - 4 } & Control & Clon 45 & Clon 75 \\
\hline Need for additional analgesia & $17 / 20$ & $10 / 20^{*}$ & $9 / 20^{*}$ \\
\hline
\end{tabular}

*There has been significant difference as compared to control group $(p=0.019)\left(X^{2}\right.$ test $)$

Intra and postoperative bradycardia was low and groups have not differed in its distribution (Table VI), but there have been significant differences in intraoperative arterial hypotension with a higher number of patients receiving the high dose as compared to control group, while patients receiving the low dose had intermediate arterial hypotension between both groups (Table VI).

Intra and postoperative side-effects were minor without significant differences among groups (Tables VII and VIII). 
Table VI - Intra and Postoperative Arterial Hypotension and Bradycardia among Groups

\begin{tabular}{lcccccc}
\hline \multirow{2}{*}{ Attributes } & \multicolumn{5}{c}{ Groups } \\
\cline { 2 - 7 } & \multicolumn{2}{c}{ Control } & \multicolumn{2}{c}{ Clon 45 } & \multicolumn{2}{c}{ Clon 75 } \\
\cline { 2 - 7 } & Intraoperative & Postoperative & Intraoperative & Postoperative & Intraoperative & Postoperative \\
\hline Arterial hypotension & $2 / 20$ & $0 / 20$ & $5 / 20$ & $1 / 20$ & $9 / 20$ * & $1 / 20$ \\
Bradycardia & $2 / 20$ & $0 / 20$ & $3 / 20$ & $1 / 20$ & $3 / 20$ & $1 / 20$ \\
\hline
\end{tabular}

*Statistically significant difference as compared to control group $(p<0.030)$ (Kruskal-Wallis)

Table VII - Intraoperative Side-Effects among Groups

\begin{tabular}{lccc}
\hline Side-Effects & \multicolumn{3}{c}{ Groups } \\
\hline Shivering & Control & Clon 45 & Clon 75 \\
Nausea & $2 / 20$ & $3 / 20$ & $2 / 20$ \\
Vomiting & $1 / 20$ & $3 / 20$ & $2 / 20$ \\
Hypoxemia & $0 / 20$ & $1 / 20$ & $0 / 20$ \\
\hline
\end{tabular}

There were no significant differences among groups $\left(\mathrm{X}^{2}\right.$ test $)$

Table VIII - Postoperative Side-Effects among Groups

\begin{tabular}{lccc}
\hline Side-Effects & \multicolumn{3}{c}{ Groups } \\
\hline Shivering & Control & Clon 45 & Clon 75 \\
Nausea & $0 / 20$ & $0 / 20$ & $0 / 20$ \\
Vomiting & $1 / 20$ & $0 / 20$ & $1 / 20$ \\
Hypoxemia & $1 / 20$ & $0 / 20$ & $0 / 20$ \\
\hline
\end{tabular}

There were no significant differences among groups $\left(\mathrm{X}^{2}\right.$ test $)$

\section{DISCUSSION}

In this study, the incidence of arterial hypotension was significant when high-level spinal anesthesia was reached with the high clonidine dose $(75 \mu \mathrm{g})$. With the low clonidine dose (45 $\mu \mathrm{g})$ the incidence of arterial hypotension was intermediate, without significant differences as compared to control group or to Clon 75 group.

In addition to peripheral and brain stem actions, spinal clonidine inhibits spinal cord sympathetic pre-ganglial neurons ${ }^{14}$. So, spinal clonidine-induced arterial hypotension intensity is related to the dermatome level in which it is administered ${ }^{15}$. In low thoracic and lumbar levels, epidural clonidine administration does not increase arterial hypotension ${ }^{15}$, but when in high thoracic levels, there is an increased incidence of such effect ${ }^{16}$. In lower spinal blocks $\left(T_{10}\right.$ to $\left.T_{6}\right)$, the incidence of arterial hypotension with 75 to $225 \mu$ g clonidine is $20 \%^{2,3,6}$. On the other hand, there is an increased incidence in patients with hypertensive diseases as compared to those with normal pressure levels, after systemic or spinal clonidine, reflecting an increase in sympathetic tone of those patients ${ }^{8}$.

In spite of the evidences relating the activation of $\alpha_{2}$-adrenergic receptors to hypotension and bradycardia caused by such receptors' agonists, one cannot exclude the participation of imidazoline $I_{1}$ receptors located in the reticular nucleus, the activation of which caused by clonidine, which is an imidazoline derivative, may determine bradycardia and arterial hypotension ${ }^{17}$.

Alpha $_{2}$-agonist-induced arterial hypotension is easily treated with $\alpha_{1}$-adrenergics such as ephedrine, which show increased vasoconstriction response in the presence of such drugs ${ }^{18}$.

The incidence of bradycardia was low, without significant differences among groups and similar to what has been already found by other authors ${ }^{6,7}$.

Our research has also shown that low clonidine dose associated to hyperbaric bupivacaine for spinal anesthesia has not interfered with major advantages obtained with this association, such as prolonged sensory block and postoperative analgesia, with lower Pain Analog Visual Scale scores and a lower number of patients needing additional analgesia in the PACU, as compared to control group.

The association has also increased motor block level 3 duration, although shorter (15\% to $25 \%$ ) as compared to doses above $75 \mu \mathrm{g}$, which is $30 \%$ to $35 \%^{8}$. It should be highlighted that motor block duration increase, which may be important in surgeries needing good muscle relaxation, is always a factor of concern and discomfort in the postoperative period. In addition, there has been no significant increase in motor blocks level 2 and 1 recovery time, with both clonidine doses.

Although already proven that clonidine prolongs spinal bupivacaine sensory, motor and analgesic block duration ${ }^{2,7}$, the mechanism of such effects is not yet well established. So, experimental ${ }^{19,21}$ and clinical ${ }^{13,22}$ studies have shown that epidural or spinal clonidine have potent analgesic action by activating gray matter $\alpha_{2}$-adrenoreceptors in spinal cord dorsal column. This antinociceptive action is present both in somatic ${ }^{19}$ and visceral pain ${ }^{23}$.

Some investigators ${ }^{7}$ also suggest a local anesthetic action unique to clonidine, which has allowed the association of the drug with local anesthetics in spinal blocks. This clonidine action, similar to local anesthetic action, and the interaction with local anesthetics has been explained by 3 possible mechanisms. First, clonidine blocks $A \delta$ and $C$ fibers as consequence of increase in potassium conductance in isolated neurons, thus intensifying local anesthetics conduction blockade ${ }^{24}$. Second, clonidine may cause local vasoconstriction, thus decreasing local anesthetic spread and removal around neural structures. This effect is medi- 
ated by drug action on post-synaptic $\alpha_{2}$-receptors although there are few evidences of this mechanism with the clinical doses used ${ }^{25}$. Third, when clonidine is associated to spinal local anesthetics, as it is the case with this study, or in peripheral blocks, it intensifies and prolongs analgesia ${ }^{26}$. Spinal clonidine is twice as potent as epidural clonidine to treat chronic pain ${ }^{27}$.

Spinal $\alpha_{2}$-adrenergic agonists may also induce analgesia by activating spinal cholinergic neurons and resulting in acethylcholine release ${ }^{28}$.

In our doses, clonidine has not induced significant sedation, which seems not to have occurred also when the drug was used in higher spinal doses $(150 \mu \mathrm{g})^{6,7}$. These results are in contrast with those obtained by the authors when oral (150 $\mu \mathrm{g})^{29,30}$ or epidural $(300 \mu \mathrm{g}){ }^{31}$ clonidine was used and the sedative effect of the drug was apparent. Locus coerulus is the major central nervous system region involved in the sedative effect of $\alpha_{2}$-adrenergic receptor agonists. Major ascending and descending adrenergic pathways are originated there. The activation of $\alpha_{2}$-receptors in this area leads to their activity suppression resulting in increased inhibitory interneurons activity increase, such as $\gamma$-aminobutyric acid pathway (GABA), which determines CNS depression ${ }^{32}$. In the conditions of this study, 45 and $75 \mu$ g clonidine associated to hyperbaric bupivacaine during high spinal anesthesia reaching dermatome $\mathrm{T}_{4}$, have maintained their major association advantages which are: analgesic block duration and postoperative analgesia. However, the highest clonidine dose $(75 \mu \mathrm{g})$ has significantly increased the incidence of arterial hypotension.

\section{REFERÊNCIAS - REFERENCES}

01. Carpenter RL, Caplan RA, Brown DL - Incidence and risk factors for side effects on spinal anesthesia. Anesthesiology 1992;76: 906-916.

02. Racle JP, Benkhadra A, Poy JY et al - Prolongation of isobaric bupivacaine spinal anesthesia with epinephrine and clonidine for hip surgery in the elderly. Anesth Analg, 1987;66:442-446.

03. Bonnet F, Cotoire P, Brun Buisson V et al - Effects of oral and subarachnoid clonidine on spinal anesthesia with bupivacaine. Reg Anesth, 1990;15:211-214.

04. Niemi L - Effects of intrathecal clonidine on duration of bupivacaine spinal anaesthesia, haemodynamics, and postoperative analgesia in patients undergoing knee arthroscopy. Acta Anaesthesiol Scand, 1994;38:724-728.

05. Klimscha W, Chiari A, Krafft P et al - Hemodynamic and analgesic effects of clonidine added repetitively to continuous epidural and spinal blocks. Anesth Analg, 1995;80:322-327.

06. Brunschwiller M, Van Gessel E, Forster A et al - Comparison of clonidine, morphine or placebo mixed with bupivacaine during continuous spinal anaesthesia. Can J Anesth, 1998;45: 735-740.

07. Fonseca NM, Aires de Oliveira C - Effects of combined clonidine and $0.5 \%$ hyperbaric bupivacaine on spinal anesthesia. $\mathrm{Br} \mathrm{J}$ Anaesthesiol, 2001;51:483-492.
08. Eisenach JC, De Kock M, Klimscha W - $\alpha_{2}$-adrenergic agonists for regional anesthesia. A clinical review of clonidine (1984-1995). Anesthesiology, 1996;85:655-674.

09. MacMillan LB, Hein L, Smith MS et al - Central hypotensive effects of the alpha (2a) - adrenergic receptor subtype. Science, 1996;273:801-803.

10. de Jonge A, Timmermans PB, van Zweiten PA - Participation cardiac presynaptic $\alpha_{2}$-adrenoceptores in the bradicardic effects of clonidine and analogues. Naunyn Schmildebergs Arch Pharmacol, 1981;137:8-12.

11. McCallum JB, Boban N, Hogan Q et al - The mechanism of $\alpha_{2}$-adrenergic inhibition of sympathetic ganglionic transmission. Anesth Analg, 1998;87:503-510.

12. Bromage PR - A comparation of the hydrochloride and carbon dioxide salts of lidocaine and prilocaine in epidural analgesia. Acta Anaesthesiol Scand, 1965;16:55-59.

13. Filos KS, Goudas LC, Patroni O et al - Hemodynamic and analgesic profile after intrathecal clonidine in humans: a dose - response study. Anesthesiology, 1994;81:591-601.

14. Guyenet PG, Cabot JB - Inhibition of sympathetic preganglionic nervous by catecholamines and clonidine: Mediation by $\alpha_{2}$-adrenergic receptor. J Neurosci, 1981;1:908-917.

15. De Koch M, Crochet B, Morimont C et al - Intravenous or epidural clonidine for intra and postoperative analgesia. Anesthesiology, 1993;79:525-531.

16. De Koch M - Site of hemodynamic effects of $\alpha_{2}$-adrenergic agonists. Anesthesiology, 1991;75:715-716.

17. Reds DJ, Regunathan S, Meely MP - Imidazoline receptors and clonidine-displacing substance in relationship to control of blood pressure, neuroprotection and adrenomedullary secretion. Am J Hypertens, 1992;5:51S-57S.

18. Goyagi T, Tanaka M, Nishikawa T - Oral clonidine premedication enhances the pressor response to ephedrine during spinal anesthesia. Anesth Analg, 1998;87:1336-1339.

19. Yaksh TL, Reddy SVR - Studies in the primate on the analgesic effects associated with intrathecal actions of opiates $\alpha_{2}$-adrenergic agonists, and baclofen. Anesthesiology, 1981; 54:451-467.

20. Eisenach JC, Dewan DM, Rose JC et al - Epidural clonidine produces antinociception, but not hypotension, in sheep. Anesthesiology, 1987;66:496-501.

21. Murata K, Nakagava I, Kumeta Y et al - Intrathecal clonidine suppresses noxiously evoked activity of spinal wide dynamic range nervous in cats. Anesth Analg, 1989;69:185-191.

22. Eisenach JC, Lysack SZ, Viscomi CM - Epidural clonidine analgesia following surgery: phase 1. Anesthesiology, 1989;71: 640-646.

23. Harada Y, Nishioka K, Kitahata LM et al - Visceral antinoceptive effects of spinal clonidine combined with morphine $\left(D \mathrm{Pen}^{2}-\right.$ $\mathrm{D}-\mathrm{Pen}^{5}$ ) enkephalen, or U50, 488H. Anesthesiology, 1995;83:344-352.

24. Butterworth JF, Strichartz GR - The $\alpha_{2}$-adrenergic agonists clonidine and guanfacine produce tonic and phasic block of conduction in rat sciatic nerve fibers. Anesth Analg, 1993;76: 295-301.

25. Nishikawa T, Dohi S - Clinical evaluation of clonidine added to lidocaine solution for epidural anesthesia. Anesthesiology, 1990;73:853-859.

26. Gaumann DM, Brunet PC, Jirounck P - Clonidine enhances the effects of lidocaine on C-fiber action potential. Anesth Analg, 1992;74:719-725.

27. Eisenach JC, Hood DD, Curry R - Relative potency of epidural to intrathecal clonidine differs between acute thermal and copsaisen-induced allodynia. Pain, 2000;84:57-64. 
28. Nagurib M, Yaksh TL - Antinoceptive effects of spinal cholinesterase inhibition and isobalographic analysis of the interaction with $\mu$ an $\alpha_{2}$-receptor systems. Anesthesiology, 1994;80:1338-1348

29. Alves TCA, Braz JCR, Ganem EM - Influence of preanesthetic clonidine on the association of sufentanil and bupivacaine in spinal anesthesia. Rev Bras Anestesiol, 1999;49:320-326.

30. Braz LG, Vianna PTG, Braz JRC et al - Sedation levels of oral preanesthetic clonidine and midazolam. Clinical and electroencephalographic bispectral analysis. Rev Bras Anestesiol, 2002;52:9-18.

31. Alves TCA, Braz JRC - Clinical evaluation of clonidine associated to ropivacaine for epidural anesthesia. Rev Bras Anestesiol, 2002;52:410-419.

32. Correa-Sales C, Rabin B, Mazze M - A hypnotic response to dexmedetomidine, an $\alpha_{2}$-agoniste is mediated in the locus coerulus in rats. Anesthesiology, 1992;76:948-952.

\section{RESUMEN}

Braz JRC, Koguti ES, Braz LG, Croitor LBJ, Navarro LHC - Efectos de la Asociación de la Clonidina a la Bupivacaína Hiperbárica en la Anestesia Subaracnoidea Alta

JUSTIFICATIVA Y OBJETIVOS: Los datos de la literatura sugieren que la clonidina, un agonista $\alpha_{2}$-adrenérgico, en asociación con la bupivacaína, en la anestesia subaracnoidea alta puede aumentar la incidencia de hipotensión y bradicardia. El objetivo de esta pesquisa fue verificar el potencial sinergismo entre dosis diferentes de clonidina, de 45 y $75 \mu \mathrm{g}, y$ bupivacaína hiperbárica en las características y en los efectos hemodinámicos del bloqueo subaracnoideo alto $\left(T_{4}\right)$.

MÉTODO: En estudio aleatorio y duplamente encubierto, fueron evaluados 60 pacientes estado físico ASA I, sometidos a cirugía del abdomen inferior y miembros inferiores. Los pacientes fueron sometidos a anestesia subaracnoidea, con bupivacaína hiperbárica a 0,5\% (17,5 mg - 3,5 ml) más la asociación de las siguientes drogas: grupo Control $(n=20)$ solución fisiológica $(0,5 \mathrm{ml})$; grupo Clon $45(n=20)$ - clonidina, en la dosis de $45 \mu \mathrm{g}(0,3 \mathrm{ml})$, asociada a la solución fisiológica $(0,2 \mathrm{ml})$; grupo Clon $75(n=20)$ - clonidina, en la dosis de $75 \mu \mathrm{g}$ $(0,5 \mathrm{ml})$. La cirugía solamente fue realizada cuando el nivel del dermátomo atingido por el bloqueo analgésico fue de $T_{4}$.

RESULTADOS: A latencia de los bloqueos analgésico y motor de la anestesia subaracnóidea no discordó significativamente entre los grupos $(p>0,05)$. Ambas dosis de clonidina prolongaron, de manera significativa, la duración del bloqueo analgésico en $T_{8}$ y del bloqueo motor grado 3 (determinado por la escala modificada de Bromage) $(p<0,05)$. La incidencia de hipotensión arterial en el per-operatorio fue evidente solamente en el grupo Clon 75 , en relación al grupo Control $(p<$ $0,05)$, con el Clon 45 presentando incidencia intermediaria entre los demás grupos. No hubo diferencia significativa entre los grupos en relación a la bradicardia $(p>0,05)$. Ambas dosis de clonidina prolongaron la analgesia pos-operatoria (intervalo de tiempo decorrido entre el bloqueo subaracnoideo y la primera solicitación de analgésico por el paciente en el pos-operatorio) $(p<0,05)$.

CONCLUSIONES: La clonidina en dosis mayor $(75 \mu \mathrm{g})$ en asociación con la bupivacaína durante anestesia subaracnoidea alta $\left(T_{4}\right)$ determina mayor incidencia de hipotensión arterial, más prolonga el bloqueo analgésico y la analgesia pos-operatoria igualmente como la menor dosis $(45 \mu \mathrm{g})$. 